\title{
Observation of a New Charmed Meson
}

H. Albrecht, U. Binder, G. Harder, I. Lembke-Koppitz, A. Philipp, W. Schmidt-Parzefall, H. Schröder, H. D. Schulz, and R. Wurth Deutsches Elektronen-Synchrotron DESY, Hamburg, Germany

J. P. Donker, A. Drescher, U. Matthiesen, H. Scheck, B. Spaan, J. Spengler, and D. Wegener Institut für Physik, Universität Dortmund, Dortmund, Federal Republic of Germany

J. C. Gabriel, K. R. Schubert, J. Stiewe, R. Waldi, and S. Weseler Institut für Hochenergiephysik, Universität Heidelberg, Heidelberg, Federal Republic of Germany

N. N. Brown, ${ }^{(a)}$ K. W. Edwards, ${ }^{(b)}$ W. R. Frisken, ${ }^{(c)}$ Ch. Fukunaga, ${ }^{(c)}$ D. J. Gilkinson, ${ }^{(d)}$ D. M. Gingrich, ${ }^{(d)}$ M. Goddard, ${ }^{(c)}$ H. Kapitza, ${ }^{(b)}$ P. C. H. Kim, ${ }^{(d)}$ R. Kutschke, ${ }^{(d)}$ D. B. MacFarlane, ${ }^{(d)}$ J. A. McKenna, ${ }^{(d)}$ K. W. McLean, ${ }^{(a)}$ A. W. Nilsson, ${ }^{(a)}$ R. S. Orr, ${ }^{(d)}$ P. Padley, ${ }^{(d)}$ P. M. Patel, ${ }^{(a)}$ J. D. Prentice, ${ }^{(d)}$ H. C. J. Seywerd, ${ }^{(d)}$ B. J. Stacey, ${ }^{(d)}$ T.-S. Yoon, ${ }^{(d)}$ and J. C. Yun ${ }^{(b)}$ Institute of Particle Physics, Montreal, Québec H3A 2A 7, Canada

R. Ammar, D. Coppage, R. Davis, S. Kanekal, and N. Kwak University of Kansas, Lawrence, Kansas 66044

G. Kernel and M. Pleško

J. Stefan Institute and Department of Physics, University of Ljubljana, Ljubljana, Yugoslavia

L. Lönsson and Y. Oku

Institute of Physics, University of Lund, Lund, Sweden

A. Babaev, M. Danilov, A. Golutvin, V. Lubimov, V. Matveev, V. Nagovitsin, V. Ryltsov, A. Semenov, V. Shevchenko, V. Soloshenko, V. Sopov, I. Tichomirov, and Yu. Zaitsev Institute of Theoretical and Experimental Physics, Moscow, Union of Soviet Socialist Republics and

R. Childers, C. W. Darden, and H. Gennow ${ }^{(\mathrm{e})}$

University of South Carolina, Columbia, South Carolina 29208

(The ARGUS Collaboration)

(Received 13 November 1985)

Using the ARGUS detector at the DORIS II $e^{+} e^{-}$storage ring at DESY, we have obtained evidence for a new charmed resonance which decays into $D^{* \pm}(2010) \pi^{\mp}$. The observed mass and width are $2420 \pm 6 \mathrm{MeV} / c^{2}$ and $70 \pm 21 \mathrm{MeV} / c^{2}$, respectively. The fragmentation function is found to be hard, as expected for a state containing a leading charm quark produced by nonresonant $e^{+} e^{-}$ annihilation.

PACS numbers: $14.40 \mathrm{Jz}, 13.25 .+\mathrm{m}, 13.65 .+\mathrm{i}$

Analogous to the excited states of mesons composed of a strange quark and lighter $u$ or $d$ quarks, excited states of charmed mesons are expected and explicit predictions for the masses of these states have been made. $^{1}$ In this Letter, we report evidence for a new charmed meson with a mass of $2420 \mathrm{MeV} / c^{2}$ decaying into $D^{*+}(2010) \pi^{-}$. (References in this paper to a specific charged state are to be interpreted as implying the charge-conjugate state also.) This is the first candidate for an orbitally excited state of the $c \bar{u}$ system.

The data presented here were collected at center-ofmass energies around $10 \mathrm{GeV}$ with the ARGUS detector at the DORIS II $e^{+} e^{-}$storage ring at DESY. A short description of the detector, trigger conditions, and multihadron selection criteria is given by Albrecht et $a l^{2}$ The event sample used for this analysis consisted of $82.4 \mathrm{pb}^{-1}$, comprising $21.6 \mathrm{pb}^{-1}$ on the $Y(1 S)$, $36.2 \mathrm{pb}^{-1}$ on the $Y(2 S), 11.5 \mathrm{pb}^{-1}$ on the $Y(4 S)$, and $13.1 \mathrm{pb}^{-1}$ obtained in nearby continuum or during scanning. Particle identification was made on the basis of measurements of specific ionization in the drift chamber and of time of flight. ${ }^{3}$

The search for excited charm states, $D^{* 0}$, has been made in the decay channel

$$
D^{* 0} \rightarrow D^{*+}(2010) \pi^{-} \text {, }
$$


where $D^{*+}(2010) \rightarrow D^{0} \pi^{+}$, and

$$
\begin{aligned}
& D^{0} \rightarrow K^{-} \pi^{+}, \\
& D^{0} \rightarrow K^{-} \pi^{+} \pi^{+} \pi^{-} .
\end{aligned}
$$

Together, these channels represent about $17 \%$ of all $D^{0}$ decays. Furthermore, the $D^{*+}(2010)$ can be easily isolated from background with good efficiency by taking advantage of the low $Q$ value for the decay $D^{*+}(2010) \rightarrow D^{0} \pi^{+}$, which results in excellent resolution for the mass difference

$$
\Delta=m\left(D^{*+}(2010)\right)-m\left(D^{0}\right) .
$$

Figure 1(a) shows the distribution of $\Delta$ for particle combinations with $m\left(K^{-} \pi^{+}\right)\left[m\left(K^{-} \pi^{+} \pi^{+} \pi^{-}\right)\right]$ lying within $\pm 45[ \pm 25] \mathrm{MeV} / c^{2}$ of the $D^{0}$ mass and with $x_{p}\left(D^{*+}(2010)\right)=p\left(D^{*+}(2010)\right) / p_{\max }>0.45$. The last requirement corresponds approximately to the region populated by $D^{* 0}$ decays with $x_{p}\left(D^{* 0}\right)>0.6$. For further analysis, a clean $D^{*+}(2010)$ sample was obtained by requiring, in addition to the cuts around the $D^{0}$ mass, that the mass difference $\Delta$ lie in the interval 144 to $147 \mathrm{MeV} / c^{2}$.

Mass combinations of selected $D^{*+}(2010)$ 's with all other $\pi^{-}$candidates in the event were then studied. Additional cuts were made on the scaled momentum of the $D^{*+}(2010) \pi^{-}$system, requiring $x_{p}\left(D^{* 0}\right)$ $>0.6$, and on the angle, $\theta$, between the $D^{*+}(2010) \pi^{-}$line of flight and the $D^{*+}(2010)$ momentum vector in the $D^{*+}(2010) \pi^{-}$rest frame, requiring $\cos \theta<0$. The first cut is motivated by the nature of charm quark fragmentation, which results in

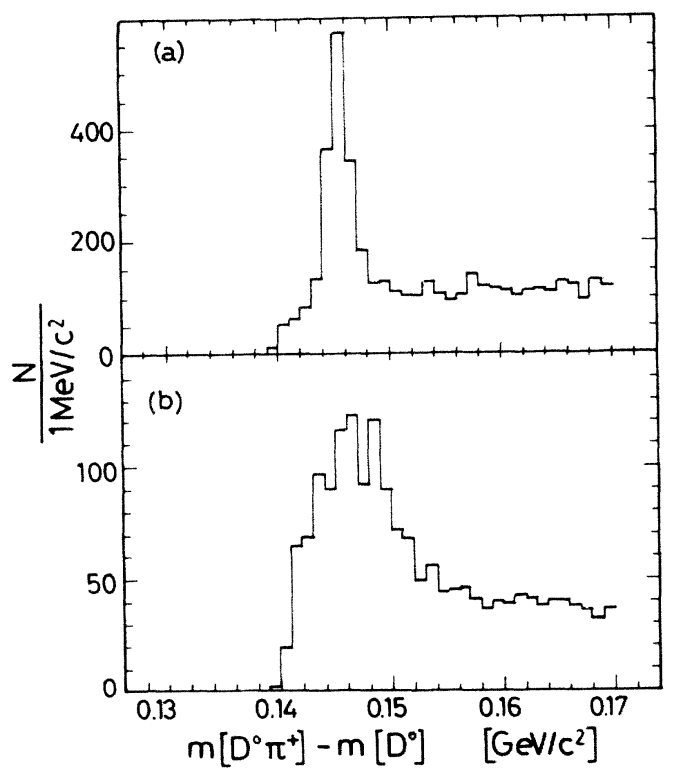

FIG. 1. (a) Distribution of the mass difference $\Delta$ for channels 1 and 2 , with $x_{p}\left(D^{*+}(2010)\right)>0.45$, not corrected for acceptance. (b) Distribution of the mass difference $\Delta$ for channel 3, with $x_{p}\left(D^{*+}(2010)\right)>0.45$, not corrected for acceptance. a hard momentum spectrum for the leading heavy meson, ${ }^{4}$ while the light hadronic background is concentrated at lower $x_{p}$. The second cut reduces the background which peaks at forward angles due to the combination of the $D^{*+}(2010)$ with random lowmomentum pions.

The mass-difference spectrum,

$$
\Delta^{*}=m\left(D^{*+}(2010) \pi^{-}\right)-m\left(D^{*+}(2010)\right),
$$

for combinations passing these cuts, is shown in Fig. 2(a). A prominent peak is seen around $410 \mathrm{MeV}$. A Breit-Wigner form for the signal, plus a threshold factor times a second-order polynomial for the background, were fitted to the mass-difference distribution, yielding the results listed in Table I. All sources of systematic error, including that introduced by the assumed mass dependence of the background, are negligible in comparison with the statistical uncertainties. Monte Carlo study shows the detector resolution to be $15 \mathrm{MeV} / \mathrm{c}^{2}$ in this mass region, while the observed width is much larger, indicating that this new state decays strongly. The statistical significance of the enhancement is 3.9 standard deviations. In the following we refer to this state as the $D^{* 0}(2420)$. It is now clear that at least part of the enhancement near 2.44 $\mathrm{GeV} / c^{2}$ in the recoil spectrum to $D^{0} \rightarrow K^{-} \pi^{+}$report-

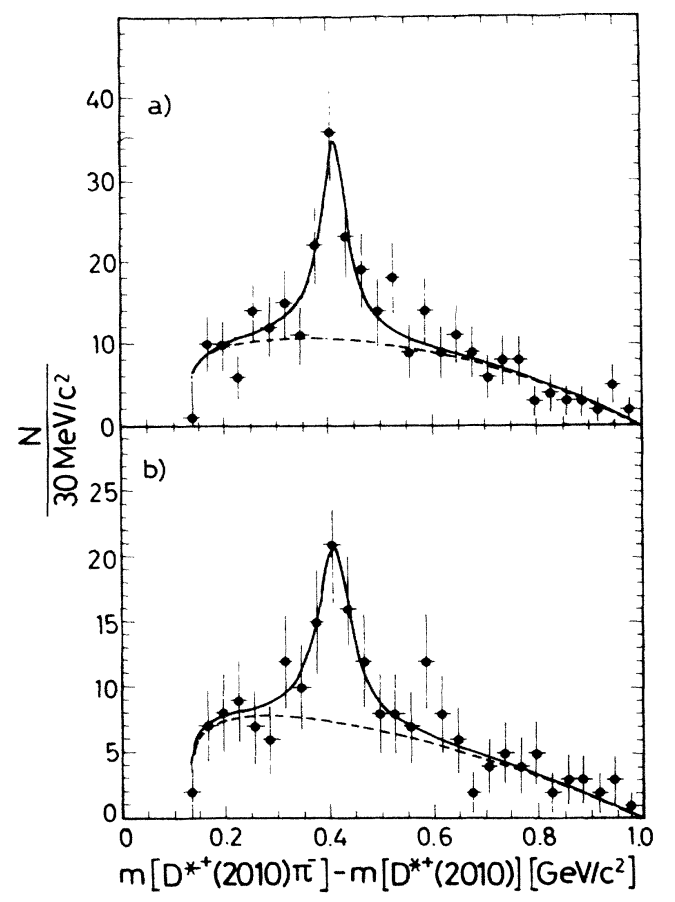

FIG. 2. (a) Distribution of the mass difference $m\left(D^{*+}(2010) \pi^{-}\right)-m\left(D^{*+}(2010)\right)$, with $x_{p}\left(D^{* 0}(2420)\right)$ $>0.6$ and $\cos \theta<0$ for channels 1 and 2. (b) Distribution of the mass difference $m\left(D^{*+}(2010) \pi^{-}\right)$ $-m\left(D^{*+}(2010)\right)$, with $x_{p}\left(D^{* 0}(2420)\right)>0.6$ and $\cos \theta<0$ for channel 3 . 
TABLE I. Properties of $D^{* 0}(2420)$ determined from fits to the distribution of mass difference, $m\left(D^{*+}(2010) \pi^{-}\right)-m\left(D^{*+}(2010)\right)$.

\begin{tabular}{|c|c|c|c|c|c|}
\hline Channel & $\begin{array}{c}\text { Mass difference } \\
\left(\mathrm{MeV} / \mathrm{c}^{2}\right)\end{array}$ & $\begin{array}{c}m\left(D^{* 0}(2420)\right) \\
\left(\mathrm{MeV} / c^{2}\right)\end{array}$ & $\begin{array}{l}\text { Full width } \Gamma \\
\left(\mathrm{MeV} / c^{2}\right)\end{array}$ & No. of events & $\chi^{2} /$ d.o.f. \\
\hline \multicolumn{6}{|l|}{$D^{0} \rightarrow K^{-} \pi^{+}$and } \\
\hline$D^{0} \rightarrow K^{-} \pi^{+} \pi^{+} \pi^{-}$ & $411 \pm 7$ & $2421 \pm 7$ & $64 \pm 26$ & $82 \pm 28$ & $20.4 / 25$ \\
\hline$D^{0} \rightarrow K^{-} \pi^{+} \pi^{0}$ & $410 \pm 11$ & $2420 \pm 11$ & $75 \pm 36$ & $52 \pm 24$ & $13.2 / 25$ \\
\hline \multicolumn{6}{|l|}{ Combined } \\
\hline result & $410 \pm 6$ & $2420 \pm 6$ & $70 \pm 21$ & $135 \pm 29$ & $16.7 / 25$ \\
\hline
\end{tabular}

ed by MARK II at the SLAC $e^{+} e^{-}$storage ring SPEAR $^{5}$ was due to production of this excited charm state.

Supporting evidence for the observation was obtained by the use of a third $D^{0}$ decay channel,

$$
D^{0} \rightarrow K^{-} \pi^{+}\left(\pi^{0}\right) .
$$

When the usual cut on the mass difference $m\left(K^{-} \pi^{+} \pi^{+}\right)-m\left(K^{-} \pi^{+}\right)$is applied, this channel produces a satellite peak in $K^{-} \pi^{+}$mass distributions, shifted to lower masses by the missing $\pi^{0}{ }^{6}$ Figure 1(b) shows the mass-difference distribution for $1540<m\left(K^{-} \pi^{+}\right)<1700 \mathrm{MeV} / c^{2}$, where again $x_{p}\left(D^{*+}(2010)\right)>0.45$ was required. Events containing $D^{*+}(2010)$ candidates decaying into this channel were selected by requiring, in addition to the noted restriction on $m\left(K^{-} \pi^{+}\right)$, that $\Delta=m\left(K^{-} \pi^{+} \pi^{+}\right)$ $-m\left(K^{-} \pi^{+}\right)<152 \mathrm{MeV} / c^{2}$. The momentum and decay-angle cuts described above were then applied to the $D^{*+}(2010) \pi^{-}$combinations. The resulting mass-difference plot for $\Delta^{*}$ is shown in Fig. 2(b). A fit to this distribution by the use of a Breit-Wigner form plus background polynomial yields the values listed in Table I. The effect of the missing $\pi^{0}$ increases the detector resolution to $25 \mathrm{MeV} / c^{2}$, but this

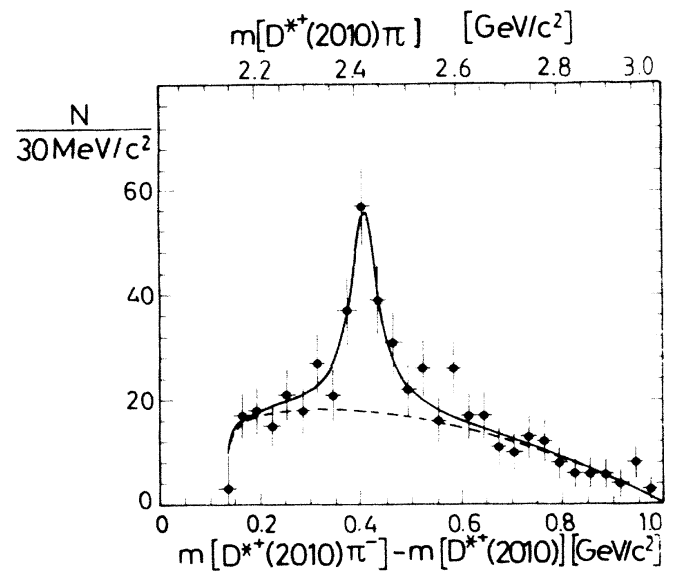

FIG. 3. Distribution of the mass difference $m\left(D^{*+}(2010) \pi^{-}\right)-m\left(D^{*+}(2010)\right)$, for the combined result shown in Figs. 2 (a) and 2 (b). is still smaller than the natural width of the state. Monte Carlo studies show that there is negligible shift in the mass difference due to the missing $\pi^{0}$. Masses and widths for the three channels are consistent: The combined significance of the effect is 4.9 standard deviations (Fig. 3).

Two different studies have been made in order to confirm that the enhancement is not an artifact of the employed kinematic selection criteria. These were made by use of (a) a sideband of the $D^{*+}(2010)$, and (b) wrong charge combinations, that is $D^{*+}(2010) \pi^{+}$. No significant enhancement was found in either approach.

The fragmentation function for the $D^{* 0}(2420)$ was extracted by the fitting of a Breit-Wigner form plus a background polynomial to the distribution of $\Delta^{*}$ selected in different $x_{p}\left(D^{* 0}(2420)\right)$ bins with $\cos \theta<0$. Only channels 1 and 2 were used for this purpose. The result, corrected for acceptance, is shown in Fig. 4, along with fits by the models of Peterson et al., ${ }^{7}$

$$
s \frac{d \sigma}{d x_{p}} \propto x_{p}^{-1}\left(1-\frac{1}{x_{p}}-\frac{\epsilon}{1-x_{p}}\right)^{-2},
$$

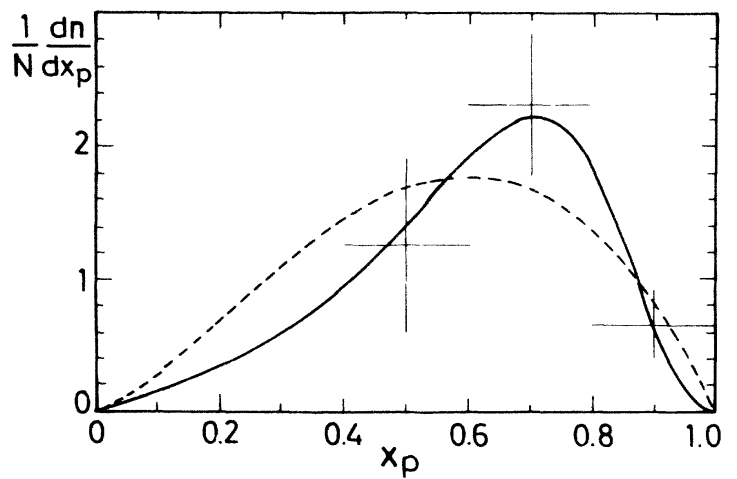

FIG. 4. Number of $D^{* 0}(2420)$ events in channels 1 and 2 as a function of $x_{p}\left(D^{* 0}(2420)\right)$, corrected for acceptance. The error bars are statistical only. The solid curve is the result of a fit to the data by the model of Peterson et al., and the dashed curve by the model of Kartvelishvili et al. The plot has been normalized so that the integral of the Peterson fit is unity. 
and Kartvelishvili et al., ${ }^{8}$

$s d \sigma / d x_{p} \propto x_{p}^{\alpha}\left(1-x_{p}\right)$.

The fitted values for the parameters of the models were $\epsilon=0.12 \pm 0.05$ with $\chi^{2}$ probability of 0.7 ( $1 \mathrm{de}$ gree of freedom), and $\alpha=1.4 \pm 0.8$ with $\chi^{2}$ probability of 0.12 . No attempt has been made to adjust these results for the effects of photon or gluon initial-state radiation. Either form describes the distribution adequately.

The production cross section for the $D^{*}(2420)$ meson decaying to $D^{*+}(2010) \pi^{-}$has been estimated by comparison with the rate observed for $D^{*+}(2010)$ production in the same data set. Channels 1 and 2 were used in determining both rates, so that common uncertainties in branching ratios cancel in the ratio. The acceptance for the $D^{*}(2420)$ due to the requirements that $\cos \theta<0$ and $x_{p}\left(D^{* 0}(2420)\right)>0.6$ was found to be $(16 \pm 5) \%$, from a detector Monte Carlo study. The error is dominated by the uncertainty in extrapolating the observed cross section to $x_{p}\left(D^{* 0}(2420)\right)=0$. The total number of observed $D^{*+}(2010)$ decays to these channels with $x_{p}\left(D^{*+}(2010)\right)>0.45$ is $1010 \pm 40$ events. Correcting this measurement also for the missing low $x_{p}\left(D^{*+}(2010)\right)$ portion of the fragmentation distribution and for acceptance, we conclude that $(24 \pm 8 \pm 8) \%$ of observed $D^{*+}(2010)$ are produced from $D^{* 0}(2420)$, where the first error is statistical and the second systematic.

The production cross section $\sigma\left(D^{* 0}(2420)\right)$ is estimated by correction for the neutral decay channel $D^{* 0}(2420) \rightarrow D^{* 0}(2008) \pi^{0}$ with the use of isospin symmetry, so that

$$
\begin{aligned}
\sigma\left(D^{* 0}(2420)\right) B\left(D^{* 0}(2420)\right. & \left.\rightarrow D^{*} \pi\right) \\
& =0.24 \sigma\left(D^{*+}(2010)\right) \times \frac{3}{2} .
\end{aligned}
$$

On the basis of results quoted in Albrecht et al., ${ }^{3}$ Hauser, ${ }^{9}$ and Wohl et al., ${ }^{10}$ we calculate that $\sigma\left(D^{*+}(2010)\right)=940 \pm 150 \pm 270$ pb at $\sqrt{s} \approx 10$ $\mathrm{GeV}$. Using this value, we find that $\sigma\left(D^{* 0}(2420)\right) B\left(D^{* 0}(2402) \rightarrow D^{*} \pi\right)=340_{-180}^{+190} \mathrm{pb}$.

The resonance reported here is most likely one of the lowest-lying orbitally excited charmed states. Of the possible assignments, ${ }^{3} P_{J}\left(0^{+}, 1^{+}, 2^{+}\right)$and ${ }^{1} P_{1}\left(1^{+}\right)$, the $0^{+}$state is excluded by parity conservation in strong decays. Predictions concerning the masses and widths of these states are complicated by the fact that the ${ }^{3} P_{1}$ and ${ }^{1} P_{1}$ states can, and probably do, mix. Also, more than one resonance may contribute to the observed signal, because the mass splittings of some of these states are less than their natural widths. However, all model calculations ${ }^{1}$ have predicted $P$ states lying within $100 \mathrm{MeV} / c^{2}$ of our observed value.

In summary, we have observed a resonance in the $D^{*+}(2010) \pi^{-}$invariant-mass distribution which we associate with a $P$ state of $c$ and $\bar{u}$ quarks. Its produc- tion and decay characteristics support some of the theoretical predictions. The mass of the object is $2420 \pm 6 \mathrm{MeV} / \mathrm{c}^{2}$, corresponding to a mass difference $\Delta m=m\left(D^{*+}(2010) \pi^{-}\right)-m\left(D^{*+}(2010)\right)$ of 410 $\pm 6 \mathrm{MeV} / \mathrm{c}^{2}$, and the width is $70 \pm 21 \mathrm{MeV} / c^{2}$.

It is a pleasure to thank E. Michel, W. Reinsch, Dr. K. Rauschnabel, Mrs. U. Djuanda, and Mrs. E. Konrad for their competent technical help in running the experiment and processing the data. We thank Dr. H. Nesemann, Dr. K. Wille, and the DORIS group for the good operation of the storage ring. The visiting groups wish to thank the DESY directorate for the support and kind hospitality extended to them. The Institute of Particle Physics is supported by the National Sciences and Engineering Research Council of Canada. This work was supported by the U.S. National Science Foundation; a University of Kansas Faculty Improvement award; the International Büro KfA, Jülich; Deutsches Electronen-Synchrotron, Hamburg; the Swedish Research Council; and the U.S. Department of Energy under Contract No. DE-AS09-80ER 10690.

(a) Also at McGill University, Montreal, Quebec H3A 2A7, Canada.

(b) Also at Carleton University, Ottawa, Ontario K1S 5B6, Canada.

(c) Also at York University, Downsview, Ontario M3J 1P3, Canada.

(d) Also at University of Toronto, Toronto, Ontario M5S 1A7, Canada.

(e) Now at the University of Stockholm, Stockholm, Sweden.

1A. De Rújula et al., Phys. Rev. D 12, 143 (1975), and Phys. Rev. Lett. 37, 398 (1976); E. Eichten et al., Phys. Rev. D 21, 203 (1980); A. B. Kaidalov, Z. Phys. C 12, 63 (1982); S. Jena, Phys. Rev. D 28, 2326 (1983); S. Godfrey and N. Isgur, Phys. Rev. D 32, 189 (1985); J. L. Basdevant and S. Boukraa, Z. Phys. C 28, 413 (1985); B. Klima and U. Maor, DESY Report No. 84-029, 1984 (to be published); B. Klima, private communication. For additional predictions concerning decay widths see V. Privman and P. Singer, Phys. Lett. 91B, 436 (1980).

${ }^{2} \mathrm{H}$. Albrecht et al. (ARGUS Collaboration), Phys. Lett. 134B, 137 (1984).

${ }^{3} \mathrm{H}$. Albrecht et al. (ARGUS Collaboration), Phys. Lett. 150B, 235 (1984).

4J. D. Bjorken, Phys. Rev. D 17, 171 (1978); M. Suzuki, Phys. Lett. 71B, 139 (1977).

${ }^{5} \mathrm{G}$. Goldhaber et al. (MARK II Collaboration), Phys. Lett. 69B, 503 (1977).

${ }^{6} \mathrm{G}$. Goldhaber, in Proceedings of the Eighteenth Rencontre de Moriond, edited by I. Tran Thanh Van (Editions Frontières, Gif-sur-Yvette, France, 1984).

${ }^{7}$ C. Peterson et al., Phys. Rev. D 27, 105 (1983).

${ }^{8}$ V. G. Kartvelishvili et al., Phys. Lett. 76B, 615 (1978).

9 J. Hauser, Ph.D. thesis, California Institute of Technology Report No. CALT-68-1275, 1985 (unpublished).

${ }^{10}$ C. G. Wohl et al. (Particle Data Group), Rev. Mod. Phys. 56, S203 (1984), and unpublished Erratum. 